\title{
ON GENERAL HYBRID CONTRACTIONS
}

\author{
S. L. SINGH and S. N. MISHRA
}

(Received 1 July 1997; revised 30 July 1998)

Communicated by J. A. Hillman

\begin{abstract}
Khan et al. have studied the existence of solutions of functional equations $f_{i} x \in S x \cap T x$ and $x=F_{i} x \in$ $S x \cap T x$ under certain nonlinear hybrid contraction and asymptotic regularity conditions, generalizing many known results on coincidences and fixed points. However, most of their main theorems admit counter examples. In this paper, we rectify these results and obtain many coincidence and fixed point theorems in a more general setting.
\end{abstract}

1991 Mathematics subject classification (Amer. Math. Soc.): primary 47H10; secondary 54H25.

Keywords and phrases: hybrid contraction, weakly commuting maps, compatible maps, weakly compatible maps.

\section{Introduction}

Nadler's multi-valued contraction theorem [10] has led to a good fixed point theory for multi-valued operators in nonlinear analysis. It has various applications inside and outside mathematics (see, for instance, $[1,19]$ ). Hybrid fixed point theory for nonlinear single-valued and multi-valued operators is a relatively recent development within the ambit of multi-valued fixed point theory (see, for instance, [2,3,5,9,11-17]). It provides techniques for solving functional inclusions and optimization problems (see [4]). Khan et al. [9] have obtained some interesting coincidence and fixed point theorems for nonlinear hybrid contractions in general settings. However, their proofs of the main theorems contain some errors, and the purpose of this paper is to improve these results under suitable conditions in a more general setting. Our results generalize, improve and unify number of results in the existing single-valued, multi-valued and hybrid fixed point theory.

(C) 1999 Australian Mathematical Society 0263-61 15/99 \$A2.00+0.00 


\section{Preliminaries}

We generally follow the definitions and notations used in [9]. Given a metric space $(X, d)$, let $(C(X), H),(C B(X), H),(C L(X), H)$ and $\left(P_{X}(X), H\right)$ denote respectively the hyper-spaces of nonempty compact, nonempty closed bounded, nonempty closed and nonempty proximal subsets of $X$, where $H$ is the Hausdorff metric induced by $d$. Notice that the hyper-space $C L(X)$ contains the other three spaces. Throughout, $D(x, A)$ will denote the ordinary distance between $x \in X$ and $A$, a nonempty subset of $X$. Also, $\mathbb{N}$ and $\mathbb{N}_{0}$ will denote respectively the sets of positive and nonnegative integers. Thus $\mathbb{N}_{0}=\mathbb{N} \cup\{0\}$. Further, for $f, g: X \rightarrow X$ and $S, T: X \rightarrow C L(X)$ we shall use the following notations for brevity.

$$
\begin{aligned}
M(S x, T y ; f, g):= & \max \{D(f x, S x), D(g y, T y), D(f x, T y), \\
& D(g y, S x), d(f x, g y)\} \\
M(S x, T y ; f):= & M(S x, T y ; f, g) \quad \text { with } f=g, \\
M(S x, y ; f):= & M(S x, T y ; f) \quad \text { with } S=T, \\
m(S x, T y ; f, g):= & \max \{D(f x, S x), D(g y, T y), \\
& \left.\frac{1}{2}[D(f x, T y)+D(g y, S x)], d(f x, g y)\right\} \\
m(S x, T y ; f):= & m(S x, T y ; f, g) \quad \text { with } f=g, \quad \text { and } \\
m(T x, y ; f):= & m(S x, T y ; f) \quad \text { with } S=T .
\end{aligned}
$$

Recall that (see [6]) mappings $f: X \rightarrow X$ and $S: X \rightarrow C L(X)$ are said to commute at a point $z \in X$ if $f S z \subset S f z ; f$ and $S$ are said to commute on $X$ if $f S x \subseteq S f x$ for all $x \in X$. We remark that weak commutativity (see [9, $14]$ ), compatibility (see $[3,8]$ ) and weak compatibility (see [12]) of maps $f$ and $S$ at a coincidence point $z$ (that is, when $f z \in S z$ ) satisfy $f S z=S f z$ (see [12, lemma]. Therefore, weak commutativity, compatibility, weak compatibility and usual commutativity of two single-valued self-maps on a metric space are equivalent at their coincidence point. However, as the following example shows, the commutativity of $f$ and $S$ at their coincidence point $z$ is indeed more general than compatibility and weak commutativity (weak compatibility) of $f$ and $S$ at $z$.

EXAMPLE 1. Let $X=[0, \infty)$ with the usual metric, $f x=3 x$ and $S x=[2+x, \infty)$. Then $f 1 \in S 1, f S 1=[9, \infty) \subset[5, \infty)=S f 1$ and $f S 1 \neq S f 1$.

\section{Main results}

Unless stated otherwise, let $\Gamma$ denote the family of maps $\phi$ from the set $\mathbb{R}^{+}$of nonnegative reals to itself such that $\phi$ is upper semi-continuous, nondecreasing and 
$\phi(t)<t$ for all $t>0$.

First we show that $[9$, Theorem 3.1] admits a counter example.

EXAMPLE 2. Let $X=\mathbb{R}^{+}$be endowed with the usual metric. Let $f, g, S, T: X \rightarrow$ $X$ be such that $f x=2 x^{2}, g x=2 x^{3}, S x=x^{2}+\frac{1}{4}$ and $T x=x^{3}+\frac{1}{4}$. Let $f_{i}$ in [9, Theorem 3.1] be such that $f_{i}=f$ if $i$ is odd and $f_{i}=g$ if $i$ is even. Evidently, for any $x, y \in X, d(S x, T y)=\left|x^{2}-y^{3}\right|=\frac{1}{2} d(f x, g y)$, that is, [9, (3.2) and (3.3)] are satisfied with $\phi(t)=q t, q=\frac{1}{2}$. Further, $S(X)=T(X)=\left[\frac{1}{4}, \infty\right) \subset X=$ $f(X)=g(X)$, that is, [9, (3.1) and (3.5)] hold, since a complete metric space is always orbitally complete.

To verify $[9,(3.4)]$, construct sequences $\left\{x_{n}\right\}$ and $\left\{y_{n}\right\}$ as considered in [9, Definition 2.1] and in the beginning of the proof of [9, Theorem 3.1]. They are defined as follows. For some $x_{0} \in X, y_{2 n}=f_{2 n} x_{2 n-1} \in S x_{2 n}$ for every $n \in \mathbb{N}, y_{2 n+1}=f_{2 n+1} x_{2 n} \in T x_{2 n+1}$ for every $n \in \mathbb{N}_{0}$.

Accordingly, for $x_{0}=\frac{1}{2}$ in our example, $\left\{x_{n}\right\}_{n=1}^{\infty}$ is given by $x_{n}=\left(\frac{1}{4}\right)^{1 / 3}$ if $n$ is odd and $x_{n}=\left(\frac{1}{4}\right)^{1 / 2}$ if $n$ is even; and $\left\{y_{n}\right\}=\left\{f x_{0}, g x_{1}, f x_{2}, g x_{3}, \ldots\right\}=\left\{\frac{1}{4}, \frac{1}{4}, \frac{1}{4}, \ldots\right\}$ which converges to $\frac{1}{4}$. Thus $[9,(3.4)]$ is satisfied for $x_{0}=\frac{1}{2}$. Therefore, all the hypotheses of [9, Theorem 3.1] are satisfied but $f, g, S$ and $T$ do not have a coincidence point. Notice, however, that $f\left(\frac{1}{2}\right)=S\left(\frac{1}{2}\right)=\frac{1}{2}$ and $g\left(4^{-1 / 3}\right)=T\left(4^{-1 / 3}\right)=\frac{1}{2}$, that is $f, S$ have a coincidence point at $x=\frac{1}{2}$ and $g$ and $T$ have a (different) coincidence at $x=4^{-1 / 3}$.

The main problem with [9, Theorem 3.1] is in the construction of the sequence $\left\{x_{n}\right\}$. Since $S(X) \cup T(X) \subset f_{i}(X)$ for each $i \in \mathbb{N}$, one cannot choose the $x_{n}$ as described in [9].

With a view of presenting a correct and more general version of [9, Theorem 3.1], we introduce the following definitions for $S, T: Y \rightarrow C L(X)$ and $f_{n}: Y \rightarrow X, n \in \mathbb{N}$. In all that follows, $Y$ is an arbitrary nonempty set.

DEFINITION 1. If for $x_{0} \in Y$, there exist sequences $\left\{x_{n}\right\}$ in $Y$ and $\left\{y_{n}\right\}$ in $X$ such that

$$
\begin{aligned}
y_{2 n+1} & =f_{2 n+1} x_{2 n+1} \in S x_{2 n} & & n \in \mathbb{N}_{0}, \\
y_{2 n} & =f_{2 n} x_{2 n} \in T x_{2 n-1}, & & n \in \mathbb{N},
\end{aligned}
$$

then $O_{f_{i}}\left(x_{0}\right)=\left\{y_{n}: n=1,2, \ldots\right\}$ is said to be the orbit for $\left(S, T ; f_{i}\right)$ at $x_{0}$. Further, $O_{f_{i}}\left(x_{0}\right)$ is called regular if

$$
d\left(y_{n}, y_{n+1}\right) \leq \begin{cases}H\left(S x_{n-1}, T x_{n}\right), & \text { if } n \text { is odd } \\ H\left(T x_{n-1}, S x_{n}\right), & \text { if } n \text { is even. }\end{cases}
$$

In the case $\left\{f_{n}\right\}=\{f, g, f, g, \ldots\}, O_{f_{i}}\left(x_{0}\right)$ and $\left(S, T ; f_{i}\right)$ may be denoted by $O_{f, g}\left(x_{0}\right)$ and $(S, T ; f, g)$ respectively. 
The above definition with $Y=X$ is the corrected version of [9, Definition 2.1]. The following definitions with $Y=X$ are [9, Definitions 2.2 and 2.3].

DEFINITION 2. If, for $x_{0} \in Y$, there exist sequences $\left\{x_{n}\right\}$ in $Y$ and $\left\{y_{n}\right\}$ in $X$ such that every Cauchy sequence of the form $O_{f_{i}}\left(x_{0}\right)$ converges in a subset $P$ of $X$, then $P$ is called $\left(S, T, f_{i}\right)$-orbitally complete with respect to $x_{0}$, or simply $\left(S, T ; f_{i}, x_{0}\right)$ orbitally complete.

DEFINITION 3. A pair $(S, T)$ is said to be asymptotically regular at $x_{0} \in Y$ if for any sequence $\left\{x_{n}\right\}$ in $Y$ and each sequence $\left\{y_{n}\right\}$ in $X$ such that $y_{n} \in S x_{n-1} \cup T x_{n-1}$, $\lim _{n}\left(y_{n}, y_{n-1}\right)=0$.

In all that follows, $C(A, f)$ stands for the set of coincidence points of the mappings $A: C \rightarrow C L(X)$ and $f: X \rightarrow X$, that is $C(A, f)=\{u: f u \in A u\}$.

The following theorem is the corrected version of $[9$, Theorem 3.1].

THEOREM 1. Let $S, T: Y \rightarrow C L(X)$ and $f_{n}: Y \rightarrow X(n \in \mathbb{N})$ be such that

(3.1) $S(Y) \subseteq f_{2 n-1}(Y), T(Y) \subseteq f_{2 n}(Y), n \in \mathbb{N}$,

(3.2) $H(S x, T y) \leq \phi\left(M\left(S x, T y ; f_{2 n}, f_{2 n-1}\right)\right)$ for each $x, y \in Y$ and $\phi \in \Gamma$,

(3.3) $\phi(t) \leq q t$ for each $t>0$ and for some fixed $q \in(0,1)$,

(3.4) there exists a point $x_{0} \in Y$ such that the pair $(S, T)$ is asymptotically regular at $x_{0}$, and

(3.5) one of $S(Y), T(Y), f_{2 i-1}(Y)$ or $f_{2 i}(Y), i \in \mathbb{N}$, is $\left(S, T ; f_{i}, x_{0}\right)$-orbitally complete.

Then:

(1a) $S$ and $f_{2 j}$ have a coincidence point for $j \in \mathbb{N}$;

(1b) $T$ and $f_{2 j-1}$ have a coincidence point for $j \in \mathbb{N}$.

Further, if $Y=X$, then

(2a) $S$ and $f_{2 j}$ have a common fixed point $f_{2 j} v$, provided $f_{2 j}\left(f_{2 j} v\right)=f_{2 j} v$ and $S$ and $f_{2 j}$ commute at $v \in C\left(S, f_{2 j}\right), j \in \mathbb{N}$;

(2b) $T$ and $f_{2 j-1}$ have a common fixed point $f_{2 j-1} w$, provided $f_{2 j-1}\left(f_{2 j-1} w\right)=$ $f_{2 j-1} w$ and $T$ and $f_{2 j-1}$ commute at $w \in C\left(T, f_{2 j-1}\right), j \in \mathbb{N}$;

(3) $S, T$ and $f_{n}(n \in \mathbb{N})$ have a common fixed point provided (2a) and (2b) both are true.

PROOF. In view of (3.1) and (3.4), for a point $x_{0} \in Y$, we can construct sequences $\left\{x_{n}\right\} \subseteq Y$ and $\left\{y_{n}\right\} \subseteq X$ such that, for each $n \in \mathbb{N}$,

$$
y_{2 n-1}=f_{2 n-1} x_{2 n-1} \in S x_{2 n-2}, \quad y_{2 n}=f_{2 n} x_{2 n} \in T x_{2 n-1}, \quad \text { and }
$$




$$
\begin{aligned}
& d\left(y_{2 n-1}, y_{2 n}\right) \leq q^{-1 / 2} H\left(S x_{2 n-2}, T x_{2 n-1}\right), \\
& d\left(y_{2 n}, y_{2 n+1}\right) \leq q^{-1 / 2} H\left(T x_{2 n-1}, S x_{2 n}\right)
\end{aligned}
$$

Then it can be easily verified that $\left\{y_{n}\right\}$ is a Cauchy sequence (see [9, proof of Theorem 3.1], [12, 13]).

Now suppose that $f_{2 i}(Y)$ is orbitally complete, $i \in \mathbb{N}$. Then the subsequence $\left\{y_{2 n}\right\}$, being contained in $f_{2 i}(Y)$, has a limit, say $u$, in $f_{2 i}(Y)$. Let $v \in f_{2 i}^{-1} u$. Thus there is a $v \in Y$ such that $f_{2 i} v=u$, and this is true for each $i \in \mathbb{N}$. Note that the subsequence $\left\{y_{2 n-1}\right\}$ also converges to $u$. Putting $x=v$ and $y=x_{2 n-1}$ in (3.2),

$$
\begin{aligned}
D\left(S v, y_{2 n}\right) \leq & H\left(S v, T x_{2 n-1}\right) \\
\leq & \phi\left(\operatorname { m a x } \left\{D\left(f_{2 n} v, S v\right), D\left(f_{2 n-1} x_{2 n-1}, T x_{2 n-1}\right), D\left(f_{2 n} v, T x_{2 n-1}\right)\right.\right. \\
& \left.\left.D\left(f_{2 n-1} x_{2 n-1}, S v\right), d\left(f_{2 n} v, f_{2 n-1} x_{2 n-1}\right)\right\}\right) \\
\leq & \phi\left(\max \left(D(u, S v), d\left(y_{2 n-1}, y_{2 n}\right), d\left(u, y_{2 n}\right), D\left(y_{2 n-1}, S v\right), d\left(u, y_{2 n-1}\right)\right\}\right) .
\end{aligned}
$$

Making $n \rightarrow \infty$, we obtain $D(S v, u) \leq \phi(D(u, S v))$, proving $u \in S v$. Thus for each $i \in \mathbb{N}, f_{i} v=u \in S v$, and this proves (1a).

Since $S(Y) \subseteq f_{2 i-1}(Y)$, there exists a $w \in Y$ such that $f_{2 i-1} w=u$, and this is true for each $i \in \mathbb{N}$, therefore, as previously, for each odd $i \in \mathbb{N}, f_{i} w=u \in T w$. This proves (1b).

If we suppose $f_{2 i-1}(Y)$ is orbitally complete $(i \in \mathbb{N}$ ), then analogous argument establishes (1a) and (1b). If $T(Y)$ (respectively $S(Y)$ ) is orbitally complete, then $u \in T(Y) \subseteq f_{2 i}(Y)$ (respectively $\left.u \in S(Y) \subseteq f_{2 i-1}(Y)\right), i \in \mathbb{N}$ and the above argument establishes (1a) and (1b).

To prove (2a), note that $Y=X, v \in C\left(S, f_{2 j}\right)$ and $u=f_{2 j} v, j \in \mathbb{N}$. From this, $f_{2 j} u=u$, and the commutativity of $S$ and $f_{2 j}(j \in \mathbb{N})$, we derive

$$
u=f_{2 j} u=f_{2 j}\left(f_{2 j} v\right) \in f_{2 j}(S v) \subseteq S\left(f_{2 j} v\right)=S u
$$

Thus $u$ is a common fixed point of $S$ and $f_{2 j}(j \in \mathbb{N})$. Similar argument yields $u=f_{2 j-1} u \in T u(j \in \mathbb{N}$, proving (2b). Now (3) is immediate.

REMARK 1. Example 2 shows that [9, Theorem 3.2] (in which $f_{i}(X)$ is misprinted as $f(X)$, see $[9,(3.7)]$ and $[9$, Theorem 3.3] are incorrect. The following are the corrected and substantially improved versions of these results.

THEOREM 2. Let $S, T: Y \rightarrow C L(X)$ and $f_{n}: Y \rightarrow X(n \in \mathbb{N})$ be such that (3.2) and the following hold.

(3.6) $\phi(t)<t$ for each $t>0$ and some $\phi \in \Gamma$, 
(3.7) there exists a point $x_{0} \in Y$ such that the orbit $O_{f_{i}}\left(x_{0}\right)$ for $\left(S, T ; f_{i}\right)$ is regular, the pair $(S, T)$ is asymptotically regular at $x_{0}$ and one of $S(Y), T(Y), f_{2 i-1}(Y)$ or $f_{2 i}(Y)(i \in \mathbb{N})$ is $\left(S, T ; f_{i}, x_{0}\right)$-orbitally complete.

Then all the conclusions of Theorem 1 are true.

THEOREM 3. Let $S, T: Y \rightarrow C(X)$ and $f_{n}: Y \rightarrow X(i \in \mathbb{N})$ be such that the conditions (3.1), (3.2) and (3.4)-(3.6) hold. Then all the conclusions of Theorem 1 are true.

PROOF. An appropriate blend of the proof of Theorem 1 works for Theorems 2 and 3. See also remarks $[9$, p. 376$]$.

REMARK 2. We now discuss another theorem of Khan et al. [9, Theorem 3.4]. Notice that [9, Theorem 3.4, condition (3.8)] and [9, Theorem 3.1, condition (3.2)] are the same. Further, [9, Theorem 3.4] is [9, Theorem 3.1] without [9, (3.3) and (3.4)]. So by Example 2, [9, Theorem 3.4] is also incorrect. Even certain special cases of [9, Theorem 3.4] admit counter examples. For instance, it can be seen using an example of Totik [18, p. 241] that [9, Theorem 3.4] with $f_{i} x=x(i \in \mathbb{N}), S, T: X \rightarrow X$ and $\phi(t)=q t,\left(\frac{1}{2}<q<1\right)$ is not true, that is $S$ and $T$ do not have a common fixed point. It seems that the authors' intention in [9, Theorem 3.4] was to present [9, Theorem $3.1]$ with $[9,(3.2)]$ replaced by the following $(*)$, since $[9,(3.4)]$ is not needed in such a situation.

(*)

$H(S x, T y) \leq \phi\left(m\left(S x, T y ; f_{i}, f_{j}\right)\right) \quad$ for $x, y \in X, i, j \in \mathbb{N}, i \neq j$, and $\phi \in \Gamma$.

However, in view of Example 2, [9, Theorem 3.4] with [9, (3.2)] being replaced by (*) remains incorrect.

The theorem we want to prove is the following:

THEOREM 4. Let $S, T: Y \rightarrow C L(X)$ and $f_{n}: Y \rightarrow X$ be such that (3.1), (3.2) with $M$ replaced by $m,(3.3)$ and (3.5) hold. Then all the conclusions of Theorem 1 are true.

PROOF. An appropriate blend of the proof of Theorem 1 works.

THEOREM 4 BIS. Theorem 4 without (3.3) and $C L(X)$ replaced by $P_{X}$.

REMARK 3. The following example shows that [9, Corollaries 3.5 and 3.6] which are intended to present important and sharpened versions of special cases of [9, Theorems 3.1 and 3.3], need some modifications in their conclusions. 
EXAMPLE 3. ([14, Example 2.4]). Let $X=[0,1]$ with the absolute value metric, and let $S x=T x=\{0,1\}, f x=1-x$ for all $x \in X$. Then $S f x=f S x$ for any $x \in X$, and $H(S x, S y)=0 \leq q d(f x, f y)$ for each $x, y \in X$ and any $q \in(0,1)$. Now it is easy to see that all the hypotheses of [9, Corollaries 3.5 and 3.6] with $S=T$ are satisfied. Evidently, $S$ and $f$ have no common fixed point. However, $S$ and $f$ have a coincidence. It may also be mentioned that this example proves that $[9$, Theorem 3.7] in its present form is not true.

Now we derive certain corollaries from our theorems, which contain and improve a number of coincidence and fixed point theorems for single-valued and multi-valued mappings in metric spaces. Corollaries 2, 2 bis and 3, 3 bis present corrected, improved and sharpened versions of $[9$, Corollaries 3.5 and 3.6$]$ respectively.

CoROllary 1. Let $S, T: Y \rightarrow C L(X)$ and $f, g: Y \rightarrow X$ be such that

(3.8) $S(Y) \subseteq g(Y)$ and $T(Y) \subseteq f(Y)$,

(3.9) $H(S x, T y) \leq q m(S x, T y ; f, g)$ for each $x, y \in Y$ and some $q \in(0,1)$, and (3.10) one of $S(Y), T(Y), f(Y)$ or $g(Y)$ is a complete subspace of $X$.

Then

(1 $\left.\mathrm{a}^{*}\right) \quad S$ and $f$ have a coincidence point $v$ in $Y$,

$\left(1 b^{*}\right) \quad T$ and $g$ have a coincidence point $w$ in $Y$.

Further, if $Y=X$, then

(2a*) S and $f$ have a common fixed point $f v$ provided $f v$ is a fixed point of $f$, and $f$ and $S$ commute at $v$,

$\left(2 b^{*}\right) \quad T$ and $g$ have a common fixed point $g w$ provided $g w$ is a fixed point of $g$ and $T$ and $g$ commute at $w$,

$\left(^{*}\right) S, T, f$ and $g$ have a common fixed point provided $\left(2 \mathrm{a}^{*}\right)$ and $\left(2 \mathrm{~b}^{*}\right)$ both are true.

The above corollary follows from Theorem 1 by noting that (3.9) implies (3.2) and (3.3), while (3.4) becomes redundant. In view of these facts, the following is a slightly more general and sharpened version of the above corollary with $f=g$.

COROLlary 2. Let $S, T: Y \rightarrow C L(X)$ and $f: Y \rightarrow X$ be such that (3.4) (with $f=g$ ) and the following hold.

(3.11) $S(Y) \cup T(Y) \subseteq f(Y)$,

(3.12) $H(S x, T y) \leq q M(S x, T y ; f)$ for each $x, y \in Y$ and some $q \in(0,1)$, and

(3.13) one of $S(Y), T(Y)$ or $f(Y)$ is $(S, T ; f)$-orbitally complete with respect to a point $x_{0}$.

Then $S, T$ and $f$ have a coincidence point $v$ in $Y$. Further, if $Y=X$, then $S, T$ and 
$f$ have a common fixed point provided $f v$ is a fixed point of $f$, and $f$ commutes with each of $S$ and $T$ at $v$.

COROLLARY 2 BIS. Corollary 2 with $Y=X, f$ continuous, and (3.13) replaced by $X$ is $(S, T ; f)$-orbitally complete with respect to a point $x_{0}$.

COROLLARY 3. Corollary 2 without (3.4) and $M$ replaced by $m$.

COROLlARY 3 BIS. Corollary 2 bis without (3.4) and $M$ replaced by $m$.

COROLlary 4. Let $T: Y \rightarrow C L(X)$ and $f: Y \rightarrow X$ be such that $T(Y) \subseteq f(Y)$ and $H(T x, T y) \leq q m(T x, y ; f)$ for each $x, y \in Y$ and some $q \in(0,1)$. If one of $T(Y)$ or $f(Y)$ is $(T, f)$-orbitally complete, then there exists a $v \in Y$ such that $f v \in T v$. Further, if $Y=X, f(f v)=f v$ and $T$ and $f$ commute at $v$, then $f v \in T f v$.

PROOF. The proof follows from Corollary 3 when $S=T$.

We remark that when all the mappings are single-valued in the above results, then, as expected, we have a unique common fixed point under relaxed conditions. For example, we present one such result which may be derived from Theorem 4 bis or can be proved on the lines of the proof of Theorem 1. Let $J$ be the family of maps $\phi$ from $\mathbb{R}^{+}$to itself such that $\phi$ is semi-continuous from the right and $\phi(t)<t(t>0)$, or nondecreasing and $\lim _{n} \phi^{n}(t)=0(t \geq 0)$ (refer to Jachymski [7] for an excellent discussion on the equivalency of these two conditions on $\phi$ ).

Corollary 5. Let $S, T, f, g: Y \rightarrow X$ be such that (3.8), (3.10) and the following hold:

$$
\begin{aligned}
& d(S x, T y) \\
& \quad \leq \phi\left(\max \left\{d(f x, S x), d(g y, T y), \frac{1}{2}[d(f x, T y)+d(g y, S x)], d(f x, g y)\right\}\right) \\
& \quad \text { for all } x, y \in Y \text { and some } \phi \in J .
\end{aligned}
$$

Then:

(i) S and $f$ have a coincidence,

(ii) $T$ and $g$ have a coincidence.

Further, if $Y=X, S f u=f S u, u \in C(S, f)$ and $T g v=g T v, v \in C(T, g)$, then $S, T, f$ and $g$ have a unique common fuxed point. 
Our results (theorems and corollaries) extend, generalize, improve and unify a number of results in the existing single-valued, multi-valued and hybrid fixed point theory (see for instance, $[3,5,7,8,10-15,17]$ and references thereof). We mention a few here. [3, Theorem 3.1] is substantially improved by Corollary 3 with $Y=X$ and $f=$ the identity mapping on X. Corollary 5 is a substantially improved version of a major theorem of Jachymski [7, Theorem 3.3], which in turn, improves a theorem of Jungck (see [8, Theorem 3.1]), Corollary 4 presents a slightly more general version of the main results of Singh and Mishra [17, Theorem 2 and Corollary 1], which, in turn, represent an improvement over Pathak's main result [12, Theorem 2], Corollary 2 is a slight improvement over the main result of Singh et al. [14, Theorem 2.1]. Several coincidence and fixed point theorems surveyed in [5] may be obtained as special cases of our results.

Finally, the following example shows that [9, Corollary 4.3] in its present form (wherein the space $X$ is complete) cannot be true unless one of the mappings in question is continuous.

EXAMPLE 4. Let $X=\left[-\frac{1}{4}, \frac{1}{4}\right]$ with the absolute value metric, and $T x=x / 3$ for all $x \in X$,

$$
f x= \begin{cases}(x-2) / 9, & x \leq 0 \\ (x+2) / 9, & x>0 .\end{cases}
$$

Then $|T x-T y| \leq \frac{1}{3}|f x-f y|$ for each $x, y \in X$ and $|T f x-f T x| \leq|T x-f x|$ for all $x \in X$, that is $T$ and $f$ are weakly commuting on $X$. Therefore, conditions [9, (4.2) and (4.3)] of the corollary in [9] are satisfied for $S=T, g=f, \phi(t)<t / 3$ but $T$ and $f$ do not have a common fixed point in $X$. Notice also that all the conditions of Corollary 5 with $Y=X, S=T$ and $g=f$ are satisfied except (3.8), since $T(X)$ is not contained in $f(X)$. Besides Corollary 5 with $Y=X$ (wherein, among other improvements, the completeness of the space has been replaced by a set of weaker conditions), several correct versions of [9, Corollary 3.4] exist in the literature (see for instance, [7, Theorem 3.3] and [8, Theorem 3.1]).

\section{Acknowledgement}

The authors express their sincere thanks to referees for their critical comments. The first author would like to express his thanks to Professor S. A. Naimpally for providing some useful references and the University of Brunei Darussalam for her hospitality while he was Visiting Professor (August 1996 - May 1997) there. The second author expresses his thanks to the University of Transkei Research Council for its grant URC $\# 4044$. 


\section{References}

[1] J. P. Aubin and I. Ekeland, Applied nonlinear analysis (Wiley, New York, 1984).

[2] J. B. Baillon and S. L. Singh, 'Nonlinear hybrid contractions on product spaces', Far East J. Math.Sci. 1 (1993), 117-127.

[3] I. Beg and A. Azam, 'Fixed points of asymptotically regular multivalued mappings', J. Austral. Math. Soc. (Series A) 53 (1992), 313-326.

[4] H. W. Corley, 'Some hybrid fixed point theorems related to optimization', J. Math. Anal. Appl. 120 (1980), 528-532.

[5] T. Hicks and B. E. Rhoades, 'Fixed points and continuity for multivalued mappings', Internat, J. Math. Math. Sci. 15 (1992), 15-30.

[6] S. Itoh and W. Takahashi, 'Single-valued mappings, multi-valued mappings and fixed point theorems', J. Math. Anal. Appl. 59 (1977), 514-521.

[7] J. Jachymski, 'Common fixed point theorems for some families of maps', Indian J. Pure Appl. Math. 25 (1994), 925-937.

[8] G. Jungck, 'Common fixed points for commuting and compatible mappings on compacta', Proc. Amer. Math. Soc. 103 (1988), 977-983.

[9] M. S. Khan, Y. J. Cho, W. T. Park and T. Mumtaz, 'Coincidence and common fixed points of hybrid contractions', J. Austral. Math. Soc. (Series A) 55 (1993), 369-385.

[10] S. B. Nadler, Jr., 'Multivalued contraction mappings', Pacific J. Math. 30 (1969), 475-488.

[11] S. A. Naimpally, S. L. Singh and J. H. M. Whitfield, 'Coincidence theorems for hybrid contractions', Math. Nachr. 127 (1986), 177-180.

[12] H. K. Pathak, 'Fixed point theorems for weak compatible multi-valued and single-valued mappings', Acta Math. Hungar. 67 (1995), 69-78.

[13] B. E. Rhoades, S. L. Singh and C. Kulshrestha, 'Coincidence theorems for some multi-valued mappings', Internat. J. Math. Math. Sci. 7 (1984), 429-434.

[14] S. L. Singh, K. S. Ha and Y. J. Cho, 'Coincidence and fixed points of nonlinear hybrid contractions', Internat. J. Math. Math. Sci. 12 (1989), 247-256.

[15] S. L. Singh and S. N. Mishra, 'Nonlinear hybrid contractions', J. Natur. Phys. Sci. 5-8 (1991-1994), 191-206.

[16] — 'Coincidence points, hybrid fixed and stationary points of orbitally weakly dissipative maps', Math. Japon. 39 (1994), 451-459.

[17] — , 'Some remarks on coincidences and fixed points', C. R. Math. Rep. Acad. Sci. Canada 18 (1996), 66-70.

[18] V. Totik, 'On two open problems of contractive mappings', Publ. Inst. Math. (Beograd) N. S. 34 (1983), 239-242.

[19] R. Wegrzyk, 'Fixed point theorems for multivalued functions and their applications to functional equations', Dissertationes Math. (Rozprawy Mat.) 201 (1982), 1-28. 
Department of Mathematics

Gurukula Kangri University

Hardwar 249404

India
Department of Mathematics University of Transkei

Umtata 5100

South Africa

e-mail: mishra@getafix.utr.ac.za 